\title{
Elementary Particles under the Lens of the Black Holes
}

\author{
George E. A. Matsas \\ Instituto de Física Teórica, Universidade Estadual Paulista \\ Rua Pamplona 145, 01405-900, São Paulo, SP, Brasil
}

Received on 8 January, 2003.

\begin{abstract}
After a brief review of the historical development and CLASSICAL properties of the BLACK HOLES, we discuss how our present knowledge of some of their QUANTUM properties shed light on the very concept of ELEMENTARY PARTICLE. As an illustration, we discuss in this context the decay of accelerated protons, which may be also relevant to astrophysics.
\end{abstract}

\section{Black Holes: Historical develop- ments}

The first black hole solution was found in 1916 by the German astrophysicist Karl Schwarzschild few months after General Relativity was formulated (and little time before his death in the Russian front). It was a static and spherically symmetric solution of the vacuum Einstein Eqs. described by the line element

$$
d s^{2}=(1-2 M / r) d t^{2}-(1-2 M / r)^{-1} d r^{2}-r^{2} d \Omega,
$$

where $M$ is the black hole mass. Notwithstanding it took many decades before the scientific community accepted that black holes were physical solutions which could be indeed realized in nature. In 1939 we can still find A. Einstein stating in the conclusions of an article [1]: "the Schwarzschild singularities do not exist in the physical reality". This was not what J. Oppenheimer and his student, H. Snyder, concluded in the same year [2], however, after analyzing the collapse of massive stars.

In 1938, J. Oppenheimer and G. Volkoff found that neutron stars had a limit for its mass beyond which they should collapse [3]. In the year after, Oppenheimer and Snyder decided to analyze it in more detail. For technical reasons, they assumed some simplifications: spherical symmetry, constant density, no rotation and no shock waves with emission of matter or radiation. Under these conditions they concluded that the collapse would lead eventually to a black hole indeed, but there remained some unclear features to be understood. In contrast to the description made by observers at rest on the surface of the star who would witness a continuous collapse towards the singularity, asymptotic observers would see the star surface like "frozen" on the event horizon. These seemingly contradictory descriptions were only reconciled after D. Finkelstein found in 1958 a coordinate system which was able to cover smoothly the internal and external regions of the black hole [4]. This conceptual step in addition with more precise numerical simulations, which were possible thanks to a better comprehension of the nuclear structure, ended up to corroborate Oppenheimer and Snyder's conclusion and bit most skepticism about the possible existence of black holes. J. Wheeler, in particular, evolved from criticizer to supporter of the black hole idea and in 1967 he introduced the denomination black hole to what was called collapsed star in the west and frozen star in the east. More than 40 years after the Schwarzschild solution was discovered, black holes were treated at least as a real possibility.

It is common to consider 1964 as the beginning of the black hole golden era. From the theoretical point of view, R. Penrose has introduced topological methods with which he was able to derive some quite general results. For example, he was able to show (under some natural assumptions in the classical realm) that black holes must have a singularity in their interior [5]. Developments in the observational domain also took place. In 1966 I. Novikov and Ya. Zel'dovich raised the possibility that there should exist binary systems formed by an ordinary star and a black hole orbiting around each other. It would be natural, thus, to expect the combined emission of X-ray and visible light from such systems since as matter is attracted by the black hole its gravitational potential would be converted into thermal energy and eventually into X-ray [6]. This turned out to be the most probable explanation for the spectrum associated with Cygnus X-1 as it became clear in 1971 with the collected data from the Uhuru satellite. It is worthwhile to notice that while the prediction that star-size black holes could be X-ray sources was confirmed only 5 years after its formulation, the explanation that radio galaxies (observed since the 30's) and quasars (observed since the 60's) were energized by the presence of super black holes had to wait more than 40 years.

Different evidences favoring the existence of black holes are mounting since then, and it is expected for soon some direct signal from a black hole event horizon. This may come as a shadow disc at the photograph plate of Sgr A* (at the center of the Milky Way, where a many-million-solarmass black hole is believed to exist) [when small enough wavelength observations become possible] or in the form of gravitational wave signals to be detected up to the 10's by the Ligo and Virgo Earth-based gravitational wave detectors or in the 20's by the Lisa space gravitational wave detector, we do not know; but what we do know is that it will be the 
confirmation of one of the greatest predictions of theoretical physics.

\section{Black Holes: CLASSICAL proper- ties}

A strongly asymptotically predictable spacetime

$$
(\mathcal{M}, g)
$$

is formally said to contain a black hole $B$ if

$$
B \equiv \mathcal{M}-J^{-}\left(\mathcal{J}^{+}\right)
$$

is not empty, i.e., if there is a region from where classical light rays cannot escape to infinity, where $J^{-}$is the causal past and $\mathcal{J}^{+}$is the future null infinity, . The event horizon of the black hole is defined as being the boundary of $B$ :

$$
H \equiv \dot{J}^{-}\left(\mathcal{J}^{+}\right) \cap \mathcal{M} \text {. }
$$

The solution discovered by Schwarzschild contains a particular kind of black hole which is static and spherically symmetric but could it exist other black holes with, let us say, more exotic forms and exquisite properties? In 1964, A. Doroshkevich, I. Novikov and Ya. Zel'dovich showed that quasi-spherically symmetric collapsing stars give rise to perfectly spherically symmetric black holes [7]. This was the prelude of a series of far-reaching theorems known as black hole no-hair theorems.

In $1967 \mathrm{~W}$. Israel derived what can be considered the first piece of this series of theorems, namely, every rotationless black hole should be spherically symmetric [8]. As a next step, it was natural, thus, to extend the analysis to rotating black holes. A solution for a rotating black hole was unveiled by R. Kerr in 1963 [9] (but only identified as so in 1965 by R. Boyer and R. Lindquist [10], B. Carter [11] and R. Penrose). At that time, it was not clear, however, if there would not exist other vacuum solutions of the Einstein Eqs. describing black holes with angular momentum. This quest was embraced by B. Carter in 1972 (with a contribution by D. Robinson) who showed that according to the vacuum Einstein Eqs. the most general black hole solution was the one given by Kerr. The event horizon of a Kerr black hole is more elongated at the equator than on the poles and the underlying geometry of a rotating black hole is richer than of a static one but still its structure remains quite simple since most properties of the original star are lost in the collapse. To put it in R. Price's words: In a star collapse process with a black hole formation, everything that can be radiated (i.e. does not satisfy some conservation law) will be radiated.

The most general formulation of the no-hair theorems associated with the electrovacuum solution of Einstein Eqs. states that black holes are completely characterized by their mass $M$, charge $Q$ and angular momentum $J$ and its geometry is described by the Kerr-Newman line element. For instance, the black hole area can be written as $(c=G=1)$

$$
A=4 \pi^{2}\left[2 M^{2}-Q^{2}+2 M \sqrt{M^{2}-Q^{2}-J^{2} / M^{2}}\right] .
$$

Thus black holes not only are probably the most exotic structures at the heavens but also one of the simplest ones as well.

\section{Black Holes: SEMICLASSICAL properties}

The beginning of the black hole semiclassical era took place in 1974. This was the summit of a number of curious events which actually began in 1971 [12]. In this year, S. Hawking showed that the total horizon area for any given set of black holes did not decrease with time. In particular, according to this theorem, black holes were indestructible. In order to derive this theorem, Hawking used some quite reasonable hypotheses (at least in the classical realm). In 1972, in analogy to the second law of thermodynamics, J. Bekenstein associated an entropy to each black hole proportional to the area of its event horizon [13]. Hawking had a strong negative reaction at first but two years later, as he analyzed the collapse of stars in the context of Quantum Field Theory in Curved Spacetimes (where positive energy conditions normally used in classical theorems are not valid), Hawking showed that black holes should radiate with a thermal spectrum [14] with temperature $\left(c=G=\hbar=k_{B}=1\right)$

$$
T=\mathcal{K} / 2 \pi
$$

(as measured by assymptotic observers), where

$$
\mathcal{K}=4 \pi \sqrt{M^{2}-Q^{2}-J^{2} / M^{2}} / A
$$

is the surface gravity. Eventually, black holes could have associated an entropy proportional to its horizon area

$$
S=k_{B} \frac{c^{3} A}{4 G \hbar}
$$

as conjectured by Bekenstein (and calculated by Hawking). This discovery opened a subarea denominated Black Hole Thermodynamics, which is presently very active because of some fundamental questions raised in connection with information theory and quantum mechanics but which will be hardly solved outside the context of a full quantum gravity theory. In Hawkings words: Holes may be black classically but are gray quantum-mechanically.

In order to understand better the Hawking effect, let us make a detour through Quantum Field Theory. It became clear since the early times of Quantum Mechanics that the no-particle state, i.e. the vacuum, has a very rich structure. Most (if not all) of its exotic properties are connected with the concept of virtual particles. Virtual particles violate the Heisenberg uncertainty principle and, thus, cannot be directly observed. Notwithstanding, they do have indirect observable consequences. Probably the most paradigmatic example of the physical consequences of the virtual particles is given by the Casimir effect.

According to the Casimir effect [15], uncharged parallel metallic plates in the vacuum experience an attractive pressure given by (see Ref. [16] for a comprehensive review and Ref. [17] for a pedagogical introduction)

$$
|F| / A=3 \pi^{2} \hbar c / 710 d^{4},
$$


where $d$ is the distance between the plates and we are discounting any gravitational effects because of the plate masses. We note that this is intrinsically a quantumrelativistic effect which would vanish for $\hbar \rightarrow 0$ and lead to nonsense results in the nonrelativistic limit $c \rightarrow \infty$. Roughly speaking, the metal plates play the role of boundaries to the virtual photons diminishing the total vacuum energy $\langle 0|\hat{H}| 0\rangle$ as the plates get closer to each other, where $\hat{H}$ is the free Hamiltonian associated with the photon field.

We already know that virtual photons feel the presence of static metallic plates but what does it happen if we consider a (nonuniformly) accelerated metallic plate in the vacuum? The metal plate will transfer energy to the virtual particles letting them real. Indeed, a photon flux will be emitted opposite to the acceleration direction while negative energy fluxes will be emitted in the acceleration direction. This is known as dynamical Casimir effect (but could be fairly called Moore effect [18]). This effect is interesting in its own right and also for being a kind of flat-spacetime analog of the Hawking effect. Here the mirror plays the role of the star, the emitted photons correspond to the Hawking radiation and the inward flux of negative energy is responsible for the black hole evaporation. The main difference here is that contrary to the mirror case, where only photons are radiated, the star collapse leads to the emission of all kind of particles. This is so because, according to the equivalence principle, all particles are coupled to gravity in the same way. What would not be easy to anticipate is that the spectrum of the emitted particles as detected by asymptotic observers can be associated to a black body. In the particular case of a static chargeless black hole, the corresponding temperature is

$$
T=\hbar c^{3} / 8 \pi k_{B} G M,
$$

where $M$ is the black hole mass. Notice the appearance of the four universal constants $c, \hbar, G, k_{B}$.

The larger the black hole, the lower the temperature and only "small-mass" particles $\left(m c^{2} \leq k_{B} T\right)$ will be likely to escape. Large-mass particles will be scattered back to the hole by the scattering potential. Notwithstanding, it is worthwhile to notice that arbitrarily large mass particles could be, in principle, observed as follows. By assuming that the evaporation process is adiabatic, the radiation temperature as measured by static observers at different Schwarzschild radial coordinates $r$ outside the black hole will differ from the one at the infinity by a red-shift factor [19], namely,

$$
T(r)=T / \sqrt{1-2 G M / r c^{2}} .
$$

Thus, the closer to the horizon the higher the temperature and the more likely to detect massive particles. However, there is no free lunch in nature: in order to probe particles with Planck mass one has to get as close to the horizon as the Planck length.

\section{Elementary particles under the lens of the black holes}

The Hawking effect connects in a nontrivial way Relativity, Quantum Mechanics, Gravity and Thermodynamics and has raised a number of different questions, some of which are still opened. Notwithstanding it became clear since 1976 after W. Unruh [20] that although static observers outside black holes detect a thermal bath of particles, free falling observers close enough to the horizon would have their detectors basically unexcited. (Here one may think of a usual 2level Unruh-DeWitt detector [21].) The explanation for this phenomenon is closely connected with previous works by S. Fulling [22] and P. Davies [23] which called attention to the fact that the particle content of a Quantum Field Theory is observer dependent. This conclusion has far-reaching implications even to Quantum Field Theory in flat spacetime. Indeed, the vacuum state as defined by inertial observers in the Minkowski space corresponds to a thermal state of all particles at temperature

$$
T=\hbar a / 2 \pi c k_{B}
$$

as detected by observers with constant proper acceleration $a$. It can be said that uniformly accelerated observers see as real those particles which inertial observers ascribe as being virtual.

It is also possible to figure out the opposite situation where particles which are unobservable to uniformly accelerated observers are observable to inertial ones. In $1991 \mathrm{~A}$. Higuchi, D. Sudarsky and the author were analyzing the following problem associated with the radiation emitted from uniformly accelerated charges. It is well known that accelerated charges radiate with respect to inertial observers and the emitted power is given by the Larmor formula [24] as (see also Ref. [25] for a deep discussion on the radiation reaction problem)

$$
W=e^{2} a^{2} / 6 \pi c^{3} .
$$

In spite of this, there was a consensus that co-accelerated observers with uniformly accelerated charges, i.e. charges with constant proper acceleration $a$, would not detect any radiation since the corresponding field is static with respect to them [26]. According to Quantum Field Theory, however, the usual classical electromagnetic radiation can be interpreted in terms of photons. So, if the co-accelerated observers did not observe any radiation, "where had the photons observed by the inertial observers gone"? The answer to this question is directly related with the fact that the elementary particle concept is observer dependent. Indeed, the emission of a finite-energy photon as seen in the inertial frame corresponds to the emission to or absorption from the Fulling-Davies-Unruh (FDU) thermal bath (in which the electron is immersed according to co-accelerated observers) of a zero-energy Rindler photon. The emission rate of finite energy photons as defined by the inertial observers and the combined emission and absorption rate of zero-energy Rindler photons as defined by the co-accelerated observers can be both written as [27] $(c, \hbar=1)$

$$
P_{k_{\perp}}(a)=\frac{e^{2}}{4 \pi^{3} a}\left|K_{1}\left(k_{\perp} / a\right)\right|^{2}
$$

where $k_{\perp}$ is the photon transverse momentum (with respect to the acceleration direction). Zero-energy Rindler photons are perfectly well defined entities since they can carry nonzero transverse momentum but cannot be detected by physical observers because they concentrate on the horizon of 
the uniformly accelerated observers [28]. From an epistemological point of view, zero-energy Rindler photons have much in common with virtual particles since although they cannot be observed they are indirectly important as a mean to explain some physical phenomena; in this case, the "disappearance" of the photons in the electron co-accelerated frame. Zero-energy particles are also important in analyzing other problems as, for instance, the response of static sources interacting with the Hawking radiation of a black hole [29].

Probably because of its non-intuitiveness the FDU effect was received with skepticism by part of the scientific community. Although the derivation of the effect is sound and the conclusion indisputable, part of the community took the position that only a "direct" observation of the effect would be convincing. Notwithstanding, this is not an easy task since no macroscopic body would resist to the typical accelerations $a$ necessary for this purpose:

$$
T /(1 K)=a /\left(2.5 \times 10^{22} \mathrm{~cm} / \mathrm{s}^{2}\right) .
$$

The strategy had to be otherwise, namely, a gedanken experiment able to make it clear that the FDU effect would be necessary for the consistency of the Quantum Field Theory itself. This was the strategy followed by D. Vanzella and the author [30] inspired by previous works [31, 27].

According to the standard model, inertial protons are stable. But this is not so for accelerated ones because of the work transferred to the proton by the external accelerating agent. As far as the proton proper acceleration satisfies $a \ll m_{n}+m_{e}+m_{\nu}-m_{p}$ the decay process will be much suppressed but for $a>m_{n}+m_{e}+m_{\nu}-m_{p}$ the weak decay channel

$$
p^{+} \rightarrow n^{0}+e^{+}+\nu
$$

will be favored up to $a \approx m_{\pi}$ after what the strong-decay channel

$$
p^{+} \rightarrow n^{0}+\pi^{+}
$$

will dominate. Recent calculations show that high-energy protons with $E \approx 10^{14} \mathrm{eV}$ under the influence of magnetic fields of $B \approx 10^{14} \mathrm{G}$ found in some pulsars should decay in a fraction of a second in laboratory time [32].

The analysis above, however, is valid for inertial observers. But how can we understand the decay process from the point of view of co-moving observers with a uniformly accelerated proton? According to these observers, in order to decay the proton must remove energy from the particles of the thermal bath in which it is immersed in its rest frame. Thus, according to the co-moving observers, the decay processes will be seen quite differently. Indeed, in the regime where the proton/neutron can be considered as unexcited/excited states of a two-level quantum mechanical system, processes (1) and (2) will be interpreted according to coaccelerated observers as

$$
\begin{aligned}
& p^{+}+e^{-} \rightarrow n^{0}+\nu \\
& p^{+}+\bar{\nu} \rightarrow n^{0}+e^{+} \\
& p^{+}+\bar{\nu}+e^{-} \rightarrow n^{0}
\end{aligned}
$$

and

$$
p^{+}+\pi^{-} \rightarrow n^{0}
$$

respectively. In particular, the correct mean lifetime is predicted in the co-accelerated frame by assuming the processes above in conjunction with the presence of the FDU thermal bath [30, 33]. Had we not taken into account the FDU thermal bath, the proton would be seemingly stable according to the co-accelerated observers (for sake of energy conservation) in contradiction with the inertial frame conclusion: The FDU effect is necessary for the consistency of Quantum Field Theory.

\section{Concluding remarks}

The overwhelming difficulty of constructing a quantum gravity theory can be illustrated by the fact that different people will give different answers to what such a theory should look like. Moreover, there is no reason to believe that the rules of quantum mechanics which are tested up to scales of $10^{-15} \mathrm{~cm}$ would not be drastically modified in the quantum gravity domain. Assuming that $c, \hbar$, and $G$ are the only fundamental constants to the quantum gravity theory, we expect that its typical effects will become obvious at the Planck scale, i.e. as soon as we accelerate elementary particles at energies of $E>M_{p} c^{2}=\sqrt{\hbar c^{5} / G}$, we are able to probe distances of $L<L_{p}=\sqrt{G \hbar / c^{3}}$, we look at processes with time scales of $T<T_{p}=\sqrt{G \hbar / c^{5}}$ or we observe structures with densities of $\rho>\rho_{p}=c^{5} / G^{2} \hbar$. In principle, these extreme situations would be likely to be realized only in singular regions, as e.g., close to the Big Bang and at black hole singularities. Unhappily the Big Bang is mostly screened by a number of effects associated with the primordial plasma (although it may be that gravitational wave detectors open a window to it) and black hole singularities are not naked. Thus, it might seem that we would be hopelessly lost from both sides: theoretically and observationally. But this is not so according to the Semiclassical Gravity theory!

If one is not allowed to visit the Chinese Imperial city, one should better wait for news just outside its limits. In our case, the Imperial city is the Quantum Gravity realm; it is forbidden to us because we do not fit into the Planck scale; and it is worthwhile to wait for news coming from it because quantum mechanical information should leak from the lock. The Hawking effect is probably the better example of how quantum gravity effects can escape towards the macroscopic domain. It might be difficult to observe the radiation emitted from large black holes since the associated temperature is very small:

$$
T /(1 K)=10^{-7} M_{\odot} / M
$$

but this is not so for the radiation emitted from smaller (primordial?) black holes. Even for large black holes, the situation is not that bad as soon as we may probe directly the region close to the horizon where the radiation temperature is very blue-shifted.

We do not know how far we will be able to go with this semiclassical approach as well as people did not know how far they were going to reach by using the semiclassical electromagnetic theory rather than QED in atomic physics; but what we do know is that every step forward in this down-up strategy will be (in principle) a long-lasting one because, after all, we are dealing with the safe side of our standard the- 
ories. Moreover because the Semiclassical Gravity is in the interface of General Relativity, Quantum Field Theory, and Thermodynamics, unexpected effects which does not have to do directly with Quantum Gravity are being unveiled. Here we have focused on the contribution of the Semiclassical Gravity Theory to the concept of elementary particle but other contributions could also be cited. Recently, Unruh has raised the very interesting possibility of mimicking the Hawking effect through Condensed Matter laboratory experiments [34]. For this purpose it is enough to arrange a compact region in a background medium (think of a spherical region in the middle of a pool) such that inside it the inward velocity of the medium is larger than the sound velocity. In this way, phonons would not be able to escape from this trapped region and we would have a sonic hole. Many (kinematical) classical and semiclassical properties of the black holes can be experimentally probed in this way. In particlular, Hawking phonon radiation is expected to be observed from sonic holes.

More embarassing than having not formulated yet the full quantum gravity theory is being aware of how much we still do not know about those theories which we thought to have mastered long ago. In this vein, quantum gravity can wait; the misteries hidden in our standard theories cannot. After all, we can always hold on V. Weisskopf words: Is it really the end of theoretical physics to get the world formula? The greatest physicists have always thought that there was one, and that everything else could be derived from it. Einstein believed it, Heisenberg believed it. I am not such a great physicist, I do not believe it... This, I think, is because nature is inexhaustible.

\section{Acknowledgments}

I am thankful to J. Castiñeiras and A. Dias for reading the manuscript and Conselho Científico e Tecnológico and Fundação de Amparo à Pesquisa do Estado de São Paulo for funding partially this work and consistent support along the years.

\section{References}

[1] A. Einstein, Ann. Math. 40, 922 (1939);

[2] J. R. Oppenheimer and H. Snyder, Phys. Rev. 56, 455 (1939).

[3] J. R. Oppenheimer and G. Volkoff, Phys. Rev. 54, 540 (1939).

[4] D. Finkelstein, Phys. Rev. 110, 965 (1958).

[5] R. Penrose, Phys. Rev. Lett. 14, 57 (1965).

[6] I. D. Novikov and Ya. B. Zel'dovich, Supp. Nuovo Cimento 4, 810 (1966); addendum 2.

[7] A. D. Doroshkevich, Ya. B. Zel'dovich, and I. D. Novikov, Sov. Phys. JETP 22, 122 (1966).

[8] W. Israel, Phys. Rev. 164, 1776 (1967).

[9] R. P. Kerr, Phys. Rev. Lett. 11, 237 (1963).
[10] R. H. Boyer and R. W. Lindquist, Jour. Math. Phys. 8, 265 (1967).

[11] B. Carter, Phys. Rev. 141, 1242 (1966).

[12] S. Hawking, Phys. Rev. Lett. 26, 1344 (1971).

[13] J. D. Bekenstein, Phys. Rev. 7, 2333 (1973).

[14] S. W. Hawking, Nature 248, 30 (1974). S. W. Hawking, Commun. Math. Phys. 43, 199 (1975).

[15] H. B. G. Casimir, Proc. Kon. Nederl. Akad. Wet. 51, 793 (1948).

[16] V. M. Mostepanenko and N. N. Trunov, The Casimir Effect and its Applications (Claredon, Oxford 1997).

[17] M. V. Cougo-Pinto, C. Farina, and A. Tort, Rev. Braz. Ens. Fis. 22, 122 (2000).

[18] G. T. Moore, J. Math. Phys. 11, 2679 (1970).

[19] R. C. Tolman, Relativity, Thermodynamics and Cosmology (Claredon Press, Oxford, 1934).

[20] W. G. Unruh, Phys. Rev. D 14, 870 (1976).

[21] B.S. DeWitt, General Relativity, eds. S.W. Hawking and W. Israel (Cambridge University Press, Cambridge, 1979).

[22] S. A. Fulling, Phys. Rev. D 7, 2850 (1973).

[23] P. C. W. Davies, J. Phys. A 8, 609 (1975).

[24] J. D. Jackson, Classical Electrodynamics, 2nd ed. (John Wiley \& Sons, New York, 1975).

[25] A. Higuchi, Phys. Rev. D 66, 105004 (2002).

[26] F. Rohrlich, Ann. Phys. (N.Y.) 22, 169 (1963). D. G. Boulware, Ann. Phys. 124, 169 (1980).

[27] A. Higuchi, G. E. A. Matsas, and D. Sudarsky, Phys. Rev. D 45, R3308 (1992); 46, 3450 (1992). H. Ren and E. J. Weinberg, Phys. Rev. D 49, 6526 (1994).

[28] J. Castiñeiras, L. C. B. Crispino, G. E. A. Matsas, and D. A. T. Vanzella, Phys. Rev. D 65, 104019 (2002).

[29] A. Higuchi, G. E. A. Matsas, and D. Sudarsky, Phys. Rev. D 56, R6071 (1997); Phys. Rev. D 58, 104021 (1998). L. C. B. Crispino, A. Higuchi, and G. E. A. Matsas, Phys. Rev. D 58, 084027 (1998); Phys. Rev. D 63, 124008 (2001). J. Castiñeiras and G. E. A. Matsas, Phys. Rev. D 60, 064001 (2000); J. Castiñeiras, I. P. Costa e Silva, and G. E. A. Matsas, Phys. Rev. D 68, 084022 (2003).

[30] G. E. A. Matsas and D. A. T. Vanzella, Phys. Rev. D 59, 094004 (1999); D. A. T. Vanzella and G. E. A. Matsas, Phys. Rev. Lett. 15, 151301 (2001).

[31] W. G. Unruh and R. M. Wald, Phys. Rev. D 29, 1047 (1984).

[32] D. A. T. Vanzella and G. E. A. Matsas, Phys. Rev. D 63, 014010 (2001).

[33] H. Suzuki and K. Yamada, to appear in Phys. Rev. D 67, 065002 (2003).

[34] W. G. Unruh, Phys. Rev. D 46, 3271 (1992). 